Article

\title{
Fault Diagnosis of Rotating Machinery Based on Multi-Sensor Signals and Median Filter Second-Order Blind Identification (MF-SOBI)
}

\author{
Feng Miao ${ }^{1, *}$, , Rongzhen Zhao ${ }^{2}$, Leilei Jia ${ }^{1}$ and Xianli Wang ${ }^{1}$ \\ 1 School of Physical and Electrical Information, Luoyang Normal University, Luoyang 471022, China; \\ jllnl@lynu.edu.cn (L.J.); wangxianli@lynu.edu.cn (X.W.) \\ 2 Key Laboratory of Digital Manufacturing Technology and Application, The Ministry of Education, Lanzhou \\ University of Technology, Lanzhou 730050, China; zhaorongzhen@lut.cn \\ * Correspondence: miaofeng@lynu.edu.cn; Tel.: +86-150-3679-1876
}

Received: 1 May 2020; Accepted: 25 May 2020; Published: 28 May 2020

\begin{abstract}
Feature extraction plays a crucial role in the diagnosis of rotating machinery faults. However, the vibration signals measured are inherently complex and non-stationary and the features of faulty signals are often submerged by noise. The principle and method of blind source separation are introduced, and we point out that the blind source separation algorithm is invalid in an environment of strong impulse noise. In order to solve the problem of fast separation of multi-sensor signals in an environment of strong impulse noise, first, the window width of the median filter (MF) is calculated according to the sampling frequency, so that the impulse noise and part of the white noise can be effectively filtered out. Next, the filtered signals are separated by the improved second-order blind identification (SOBI) algorithm. At the same time, the method is tested on the strong pulse background noise and rub impact dataset. The results show that this method has higher efficiency and accuracy than the direct separation method. It is possible to apply the method to real-time signal analysis due to its speed and efficiency.
\end{abstract}

Keywords: fault diagnosis; vibration signal; rotating machinery; rotor

\section{Introduction}

Rotating machinery is a kind of widely used power equipment, so it is of great significance to implement intelligent operation and maintenance management [1,2]. However, due to the complex structure and dynamic operating environment, the vibration of such equipment generally presents strong nonlinear and background noise characteristics [3,4]. For many years, only the local vibration signals collected by a single sensor have been used to solve the problem of fault identification of rotating machinery system, which has led to obvious difficulties. Thus, we should make full use of a series of sensors arranged at several key sections of the rotating machinery, and implement intelligent fault decision-making technology based on as much information as possible. This view has gained consensus on the research prospect of industrial big data technology [5]. Obviously, the more sensors that collect fault information, the more dimensions the fault feature dataset will have. Therefore, in the process of researching the intelligent operation and maintenance of rotating machinery, a major question is how to effectively extract the sensitive quantitative features of its operational state from the nonlinear, strong noise, high-dimensional vibration signal fault feature dataset, which has significance for the development of intelligent decision-making technology driven by big data.

The purpose of a vibration signal analysis of rotating machinery is to extract the operational information of the key rotating parts (e.g., gears, bearings, and rotors), and effective signal processing and feature extraction are key to completing the condition monitoring and fault diagnosis of rotating 
machinery. Therefore, more accurate and comprehensive extraction of vibration signal characteristics has been the pursuit of researchers in this field. The methods of feature extraction of vibration signal include empirical mode decomposition (EMD) [6,7], minimum entropy deconvolution [8,9], an adaptive filter [10,11], matching tracking [12,13], mathematical morphology analysis [14-16], cyclostationary signal analysis [17,18], a Wiener filter [19,20], wavelet transform [21-23], a Kalman filter [24,25], and stochastic resonance $[26,27]$-all of which help with the development of mechanical fault diagnosis. The above methods can effectively eliminate background noise and interference components, and extract a fault signal in a specific environment, but are not suitable for complex interference situations. In particular, when the interference signal in the vibration signal of rotating machinery is similar to the fault signal, it is more difficult to identify and eliminate the interference components and extract the fault signal with the above methods. Blind source separation (BSS) technology [28] can separate multiple aliased signals without the influence of time-frequency overlap of source signals. At the same time, the separation signal will not lose the weak signal characteristic information in the vibration signal of rotating machinery.

There have been many effective blind source separation algorithms with different characteristics, including the fast fixed-point algorithm [3], natural gradient algorithm [29], Equivariant Adaptive Separation via Independence (EASI) algorithm [30], and Joint Approximation Diagonalization of Eigen-matrices (JADE) algorithm [31]. These have been widely used for fault diagnosis [32,33], image processing [34], speech recognition [35], earthquake prediction [36], and other fields. Lu et al. [37] proposed a source contribution quantitative estimation method based on underdetermined blind source separation to identify the major vibration and radiation noise. Belaid et al. [38] presented a new multiscale decomposition algorithm that enables the blind separation of convolutely mixed images. He et al. [39] proposed an improved underdetermined blind source separation algorithm based on sparse component analysis (SCA) and applied on the sampled pressure pulsation signal to obtain separated signals. Zhang et al. [40] proposed a universal single-channel blind source separation method based on a combination of ensemble empirical mode decomposition and independent component analysis, which is suitable for autonomous underwater vehicles in time-varying ocean currents. These algorithms usually show good separation performance when separating noiseless mixed signals, but when separating strong noiseless mixed signals, there could be a large error-especially in the case of low signal to noise ratio (SNR), there will be a completely wrong conclusion, because these algorithms are based on the noiseless interference model. In rotating machinery operation, the signals measured by sensors inevitably contain noise. If the above blind source separation algorithm is directly used to separate the mixed vibration signal, many errors may be produced, or the wrong conclusions may be drawn.

Therefore, a fault diagnosis method for rotating machinery is developed based on a combination of improved median filter (MF) and second-order blind identification (SOBI). Firstly, the window width of the median filter is calculated according to the sampling frequency, so that the impulse noise and part of the white noise can be effectively filtered out. Secondly, the improved SOBI algorithm is used to separate the signal after noise reduction. Finally, the method is tested on strong pulse background noise and the rub impact dataset, and the fault feature signal is effectively separated.

\section{Model of Fault Diagnosis System}

In the condition monitoring and fault diagnosis of rotating machinery, the signal measured by the sensor is usually a linear mixture of multiple original signals. The mixing process is shown in Figure 1.

Suppose the signal vector observed from $m$ sensors is $x(t)=\left[x_{1}(t) x_{2}(t) \cdots x_{m}(t)\right]^{T}$, and the vibration signal vector of $n$ signal sources is $s(t)=\left[s_{1}(t) s_{2}(t) \cdots s_{n}(t)\right]^{T}$, where $x_{i}(t)$ represents the signal value observed by the $i(i=1,2, \cdots, m)$ sensors at time $t$, while $s_{i}(t)$ represents the vibration 
signal value of the $i(i=1,2, \cdots, n)$ signal sources at time $t$. The output signal of each sensor can be expressed as follows [41]:

$$
x_{i}=\sum_{j=1}^{n} a_{i j} s_{j}(t)+v_{i}(t) ; i=1,2,3, \cdots, m
$$

where $a_{i j}$ is the mixing coefficient, and $v_{i}(t)$ is the noise signal.

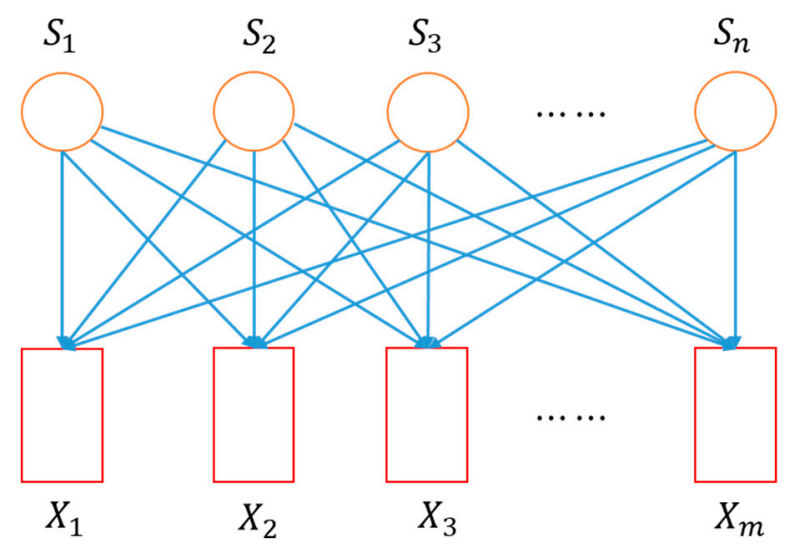

Figure 1. Diagram of multisource multi-sensor testing procedure.

Its matrix form can be expressed as follows:

$$
\left(\begin{array}{c}
x_{1} \\
x_{2} \\
x_{3} \\
\vdots \\
x_{m}
\end{array}\right)=\left(\begin{array}{ccc}
a_{11} & \cdots & a_{1 n} \\
\vdots & \vdots & \vdots \\
a_{m 1} & \cdots & a_{m n}
\end{array}\right)\left(\begin{array}{c}
s_{1} \\
s_{2} \\
s_{3} \\
\vdots \\
s_{n}
\end{array}\right)+\left(\begin{array}{c}
v_{1} \\
v_{2} \\
v_{3} \\
\vdots \\
v_{n}
\end{array}\right)
$$

which is

$$
x(t)=A s(t)+v(t),
$$

where $A \in R^{m \times n}$ is an unknown full-rank mixed matrix; $s(t)$ is an $n$-dimensional source vector; $v(t)$ is an additive noise vector, and its statistics are independent.

The purpose of blind source separation is to find a separation matrix $W$, so that the result of Equation (4) is the optimal estimate of $s(t)$ :

$$
y(t)=W x(t)
$$
formula:

To separate the source signal is to find the separation matrix $U$ and make it meet the following

$$
\widehat{S}(t)=U X(t)=U A \widetilde{S}(t),
$$

if

$$
U A=I .
$$

If $I$ is a unit matrix, then $\widehat{S}(t)$ is an effective separation of $\widetilde{S}(t)$.

However, the structure of key parts of the rotating machinery is complex: there are many sources of vibration excitation, the actual propagation path of the vibration is complicated, the equipment interferes with each other, and the environmental interference is noisy. The measured signal is often 
a result of multiple vibration sources overlapping with each other. In a linear convolutional hybrid model, the observation vector can be expressed as follows:

$$
x(t)=A(t) * s(t)+v(t) .
$$

The convolution in the time domain is equal to the product in the frequency domain, and the convolution mixture in the time domain is converted into an instantaneous mixture in the frequency domain. Its model can be expressed as follows:

$$
x(f)=A(f) s(f)+v(f) .
$$

The two are unified in form. Therefore, no matter how the signals are mixed, they can be processed in the same way.

In this article, unless otherwise stated, the following assumptions are made:

(1) The mixed matrix $A \in R^{m \times n}$ is full rank;

(2) The source signal is a zero-mean, spatially uncorrelated, but time-correlated signal;

(3) The source signal is a stationary process, i.e.,

$$
E\left\{s(t+\tau) s^{T}(t)\right\}=\operatorname{diag}\left[\rho_{1}(\tau), \cdots, \rho_{n}(\tau)\right] .
$$

\section{The Median Filter Method}

\subsection{Principle of Median Filter}

A median filter is a kind of nonlinear filter technology that has good edge preserving characteristics and the ability of suppressing impulse noise [8]. This method is essentially a window filter. The filtering operation is to scan the sample data by sliding a fixed length window, and replace the data in the center of the current window with the median of the data in the window [42]. With the end of the window moving, the filtering process of the whole sample signal will be completed. Since only one signal dimension is involved in the vibration signal analysis in this paper, only one dimension of the discrete median filtering principle is discussed. Let the discrete sampling sequence of signal $x(t)$ be $x(n)(n=1,2,3 \cdots, n)$ and take the filtering window with a length of $L_{d}=2 d+1$ (where $d$ is a positive integer) to conduct median filtering for this signal sequence. At the $n$th time, the data column in the window is represented as $W_{d}=\{x(n-d), \cdots, x(n), \cdots, x(n+d)\}$, and $n-d \geq 1$, $n+d \leq N$. The number of $2 d+1$ in $W_{d}$ is arranged in the order of small to large, and the intermediate value $y(n)$ is taken to replace the original $x(n)$ that is, the filtering task of a data point of the signal is completed $[43,44]$. The mathematical expression of this process is:

$$
y(n)=\operatorname{Med}[x(n-d), \cdots, x(n), \cdots, x(n+d)]
$$

where $\operatorname{Med}[]$ is the median of all numbers in the window.

The principle of the median filter is simple, it is easy to create its algorithm program with a computer, and the impulse noise under half window width can basically be eliminated. Therefore, as long as an appropriate window width is set, the median filter can effectively reduce the impulse noise in the vibration signal, but because of the characteristics of the filtering method itself, it cannot filter out the white noise.

\subsection{Improved Method of Median Filter}

The key problem with the median filtering method is determining the filter window width according to the signal characteristics. On the one hand, the window should not be too wide, or the details of useful signals will be lost; on the other hand, the window should not be too narrow, or too 
much impulse noise will remain. In order to filter out impulse noise without losing useful signal, the window width should be double that of the pulse width. If the sampling interval of vibration signal is $T_{s}$ and the duration of impulse noise is $L_{s}$, the reasonable window width $L_{d}$ can be expressed as follows:

$$
L_{d}=2 L_{s} F_{s}
$$

where $F_{s}=1 / T$ is the sampling frequency. According to [16], the impulse noise is mainly continuous: $(5.36 \pm 2.48) \times 10^{-4} \mathrm{~s}$. For the convenience of calculation and without loss of useful signal, $L_{s}=5.00 \times 10^{-4} \mathrm{~s}$ is assumed. It can be seen from Equation (11) that the window width is adaptively adjusted with $F_{s}$, which is more conducive to eliminating impulse noise and retaining useful signals.

\section{The Fast Second-Order Blind Identification (SOBI) Method for Diagonalization of Average Matrix}

The second-order statistical method does not require the non-Gaussian assumptions applied in the higher-order statistical method $[43,44]$. However, because the second-order statistical method is usually a joint diagonalization of a set of autocorrelation matrices, the dimension of the autocorrelation matrix increases with the number of sensors, so when the number of sources is quite large, the calculation of the BSS method is more difficult. Therefore, a simple and effective SOBI based BSS method is needed.

\subsection{The SOBI Method for Joint Approximation Diagonalization}

\subsubsection{The Joint Approximation Diagonalization SOBI Method}

Methods for the blind separation of second-order statistics generally include preprocessing data, calculating second-order statistics, calculating diagonalization matrices, and obtaining source and mixed process estimates.

\section{(1) Data Preprocessing}

The most common preprocessing step in the second-order blind separation method is whitening. When the number of source signals and the number of sensors is the same, no whitening treatment is required. However, the whitening preprocessing can remove the spatial correlation. When the number of source signals and sensors are not the same, the whitening process can estimate the number of sources, and, at the same time, eliminate the effect of additive noise on the signal. Belouchrani and Cichocki [45] improved the algorithm by using robust orthogonalization as a preprocessing step, under the condition that the number of observations was greater than the number of sources.

(2) Calculate Second-Order Statistics

For colored sources with different power spectrums, a delay covariance matrix is used:

$$
M_{i}=R\left(\tau_{i}\right)=E\left\{x(t+\tau) x^{*}(t)\right\}
$$

(3) Joint Matrix Diagonalization to Obtain Unitary Matrix

The goal of joint diagonalization is to find the orthogonal matrix $U$, enabling a group of matrices to be diagonalized, so

$$
M_{i}=U D_{i} U^{T} \quad(i=1,2, \cdots, K)
$$

In this formula, $M_{i}$ is the delay covariance, and $D_{i}$ is the real diagonal matrix.

\subsubsection{Algorithm Steps for Joint Approximate Diagonalization SOBI Method Implementation}

The implementation algorithm flow of the SOBI method is as follows: 
Step 1: Estimate the sample covariance matrix $\widehat{R}(0)$ from $T$ sample data; use $\lambda_{1}, \cdots, \lambda_{n}$ to represent the $n$ largest eigenvalues, and have $h_{1}, \cdots, h_{n}$ as the corresponding eigenvectors. Under the assumption of white noise, the noise variance estimate $\bar{\sigma}^{2}$ is the average of $m-n$ minimum eigenvalues of $\widehat{R}(0)$. In the ideal case without noise, the last $m-n$ singular values of $\widehat{R}(0)$ are equal to 0 . Therefore, the noise variance $\bar{\sigma}^{2}$ is 0 .

Step 2: Obtain the whitening signal $z(t)=\left[z_{1}(t), \cdots, z_{n}(t)\right]^{T}$, where $z_{i}(t)=\left(\lambda_{i}-\bar{\sigma}^{2}\right)^{-\frac{1}{2}} h_{i}^{*} x(t)$; $1 \leq i \leq n$. Using $\widehat{W}=\left[\left(\lambda_{1}-\bar{\sigma}^{2}\right)^{-\frac{1}{2}} h_{1}, \cdots,\left(\lambda_{n}-\bar{\sigma}^{2}\right)^{-\frac{1}{2}} h_{n}\right]^{H}$ forms a whitening matrix.

Step 3: Fix a set of delays $\tau \in\left\{\tau_{j} \mid j=1, \cdots, K\right\}$, estimate the covariance matrix $\widehat{R}(\tau)$ of the samples, and obtain the whitened signal $\underline{\widehat{R}}(\tau)$.

Step 4: Get the approximate diagonalizer $\widehat{U}$ of the set $\left\{\underline{\underline{R}}\left(\tau_{j}\right) \mid j=1, \cdots, K\right\}$.

Step 5: Calculate the estimate of the source signal $\widehat{s}(t)=\widehat{U}^{H} \widehat{W} x(t)$, and the mixed matrix $A$ estimates $\widehat{A}=\widehat{U}^{*} \widehat{W}$.

4.2. The Fast SOBI Method Based on Diagonalization of the Average Matrix

\subsubsection{Method Principle}

The structure of the correlation matrix with different time delays observed by the sensor is as follows:

$$
\begin{gathered}
R(0)=E\left(x(t) x^{*}(t)\right) \\
R(0)=E\left(x(t) x^{*}(t)\right), \forall \tau \neq 0
\end{gathered}
$$

Spatially whiten the correlation matrix $R(0)$ :

$$
\underline{R}(0)=\widehat{W} R(0) \widehat{W}^{H},
$$

where $\widehat{W}$ is the whitening matrix. The whitening correlation matrix $\underline{R}(\tau)$ with different time delays is defined as

$$
\underline{R}(\tau)=\widehat{W} R(\tau) \widehat{W}^{H} \quad \forall \tau \neq 0
$$

The whitened correlation matrix forms a joint matrix $M=\left\{M_{1}, \cdots, M_{K}\right\}$, where

$$
\begin{aligned}
M_{1}=\underline{R}\left(\tau_{1}\right) & =\widehat{W} R\left(\tau_{1}\right) \widehat{W}^{H} \\
& \vdots \\
M_{K}=\underline{R}\left(\tau_{K}\right) & =\widehat{W} R\left(\tau_{K}\right) \widehat{W}^{H}
\end{aligned}
$$

Due to

$$
\begin{aligned}
& \sum_{i \neq j}\left(V^{H}\left(M_{1}+\cdots+M_{K}\right) V\right) \\
= & \sum_{i \neq j}\left(V^{H} M_{1} V+\cdots+V^{H} M_{K} V\right) \\
= & \sum_{i \neq j} V^{H} M_{1} V+\cdots+\sum_{i \neq j} V^{H} M_{K} V
\end{aligned}
$$

using the general diagonalization criterion

$$
C(M, V)=\sum_{k=1}^{K} \sum_{i \neq j}\left(\left(V^{H} M_{k} V\right)_{i j}\right),
$$


we can get

$$
\begin{gathered}
C(M, V)=\sum_{i \neq j}\left(\left(V^{H} M_{1} V\right)_{i j}+\cdots+\left(V^{H} M_{k} V\right)_{i j}\right) \\
=\sum_{i \neq j}\left(V^{H}\left(M_{1}+\cdots+M_{k}\right) V\right) \\
=\sum_{i \neq j}\left(V^{H} \sum_{k=1}^{K}\left(M_{i}\right) V\right)
\end{gathered}
$$

Divide both sides by $K$ and get the new diagonalization criterion:

$$
\begin{aligned}
C_{\text {new }}(M, V) & =\sum_{i \neq j}\left(V^{H}\left(\frac{1}{K} \sum_{k=1}^{K} M_{i}\right) V\right) \\
& =\sum_{i \neq j}\left(V^{H} \text { mean }(M) V\right)
\end{aligned}
$$

The improved diagonalization method is based on the following optimization problems:

$$
\min _{V \in R^{n \times n}} \sum_{i \neq j}\left(V^{H} \operatorname{mean}(M) V\right) .
$$

The joint diagonalization of the matrix is transformed into the diagonalization problem of the average matrix, which greatly simplifies the algorithm. The average matrix reflects the average feature structure of the matrix, which will inevitably cause the loss of some detailed features; this will affect the separation accuracy to some extent, but it can be seen from the subsequent experiments that this simplification does not seriously affect the separation effect. However, the separation speed was significantly improved.

\subsubsection{Implementation Algorithm Steps of Improved MF-SOBI Method}

The implementation algorithm flow of the improved MF-SOBI method is as follows:

Step 1: the improved median filtering method in Section 3.2 is used to filter the sampled signal.

Step 2: estimate the filtered sample covariance matrix $\widehat{R}(1)$ from the T sample data. Singular value decomposition of $\widehat{R}(1)$ is performed to obtain a whitening matrix $\widehat{W}$. Let $\lambda_{1}, \cdots, \lambda_{n}$ represent the $n$ largest eigenvalues, and $h_{1}, \cdots, h_{n}$ the corresponding eigenvector.

Step 3: under the assumption of noise, the estimate of noise variance $\bar{\sigma}^{2}$ is the average of $m-n$ minimum eigenvalues of $\widehat{R}(1)$. In the ideal case of no noise or white noise, the last $m-n$ singular values of $R$ are equal to 0 .

Step 4: fix a set of delay $\tau \in\left\{\tau_{j} \mid j=1, \cdots, K\right\}$, estimate the sample covariance matrix $\widehat{R}(\tau)$, and calculate $\left\{\underline{\widehat{R}}\left(\tau_{j}\right) \mid j=1, \cdots, K\right\}$.

Step 5: obtain the approximate diagonalizer $\widehat{U}$ or diagonalization matrix $\widehat{V}$ of the average matrix $\frac{1}{K} \sum_{k=1}^{K} \widehat{\widehat{R}}\left(\tau_{j}\right)$ of the set $\left\{\underline{\widehat{R}}\left(\tau_{j}\right) \mid j=1, \cdots, K\right\}$.

Step 6: the estimation of the source signal is $\widehat{s}(t)=\widehat{U}^{H} \widehat{W} x(t)$ or $\widehat{s}(t)=V^{-1} \widehat{W} x(t)$, and the estimation of the mixed matrix $A$ is $A=\widehat{W}^{*} \widehat{V}$.

\section{Simulation Test Research}

\subsection{Evaluation Index of Method Separation Performance}

(1) Performance Index (PI) 
To measure the effectiveness of the method, a performance indicator is used to measure the separation performance [46], which is defined as

$$
P I=\frac{1}{n(n-1)} \sum_{i=1}^{n}\left\{\left(\sum_{k=1}^{n} \frac{\left|g_{i k}\right|}{\max _{j}\left|g_{i j}\right|}-1\right)+\left(\sum_{k=1}^{n} \frac{\left|g_{k i}\right|}{\max _{j}\left|g_{j i}\right|}-1\right)\right\}
$$

In the formula, $g_{i k}$ is the $(i, j)$ th element of the system matrix $G=\widehat{U}^{H} \widehat{W} A$, and $\max _{j}\left|g_{i j}\right|$ represents the maximum value of the element absolute value of the vector in the $i$ th row of G. Similarly, $\max _{j}\left|g_{j i}\right|$ represents the maximum value of the absolute value of the element in the vector in the ith column of $G$. The smaller the PI, the better the separation effect. When complete separation is achieved, the performance index is 0 .

(2) Residual Cross-Talking Error (RCTE)

The residual cross-talking error (RCTE) between the separated signal and the source signal also reflects the similarity between the separated signal and the source signal. Generally, when the RCTE value reaches $-20 \mathrm{~dB}$, it can be considered to achieve a good separation effect:

$$
e_{R C T E}\left(s_{i}, x_{i}\right)=10 \lg \left(\frac{E\left[\left(s_{i}-x_{i}\right)^{2}\right]}{E\left(x_{i}^{2}\right)}\right)
$$

where $s_{i}$ is the $i$ th source signal and $x_{i}$ is the $i$ th comparison signal.

\subsection{Simulation Study on Separation of Mixed Signals with Different Frequency Carriers}

In the simulation experiment, the vibration of the rotor system is mainly simulated. Rotating parts of the rotor system include the rotor, bearing, and other rotating parts. The vibration signal of the rotor system can be determined from the sinusoidal signal of each frequency and each resonance frequency. The simulation signal can be expressed as

$$
\begin{gathered}
s_{1}=\sin (100 \pi t) \\
s_{2}=\cos (200 \pi t)+\sin (300 \pi t) \\
s_{3}: \text { random noise }
\end{gathered}
$$

The randomly generated mixed matrix is as follows:

$$
A=\left[\begin{array}{lll}
0.4 & 0.8 & 0.3 \\
0.6 & 0.1 & 0.9 \\
0.8 & 0.5 & 0.4
\end{array}\right]
$$

Assume that the rotor rub impact fault frequency is a mixed signal of $100 \mathrm{~Hz}$ and $150 \mathrm{~Hz}$, and other source signals are composed of a vibration signal with a base frequency of $50 \mathrm{~Hz}$ and Gaussian white noise. The source signal waveform obtained is shown in Figure 2. 

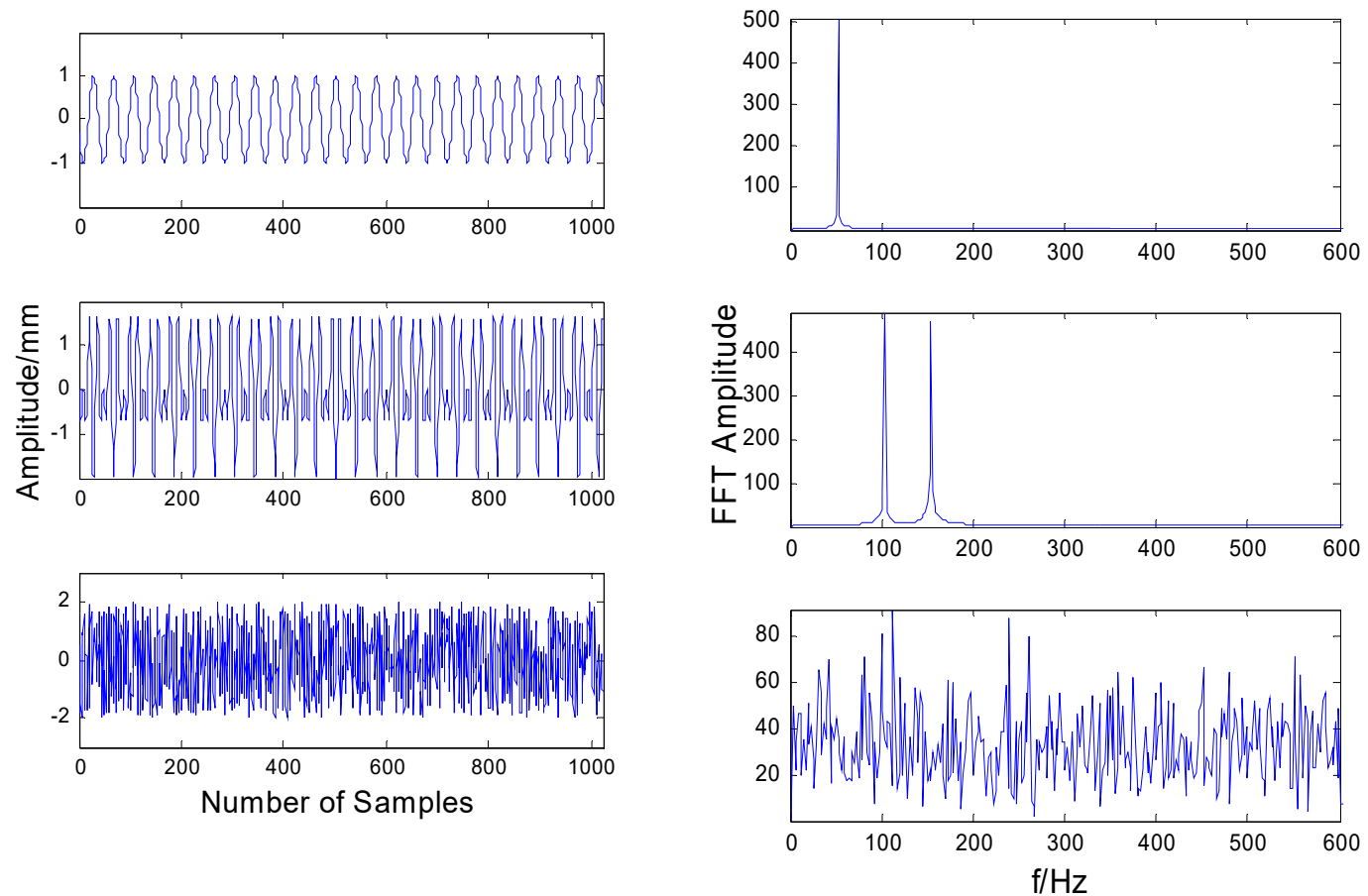

(a) The time domain signals.

(b) The frequency domain signals.

Figure 2. The time-frequency waveform of source signal.

The source signal is randomly and linearly mixed, and the mixing matrix is $A$. The mixed signal is as shown in Figure 3.
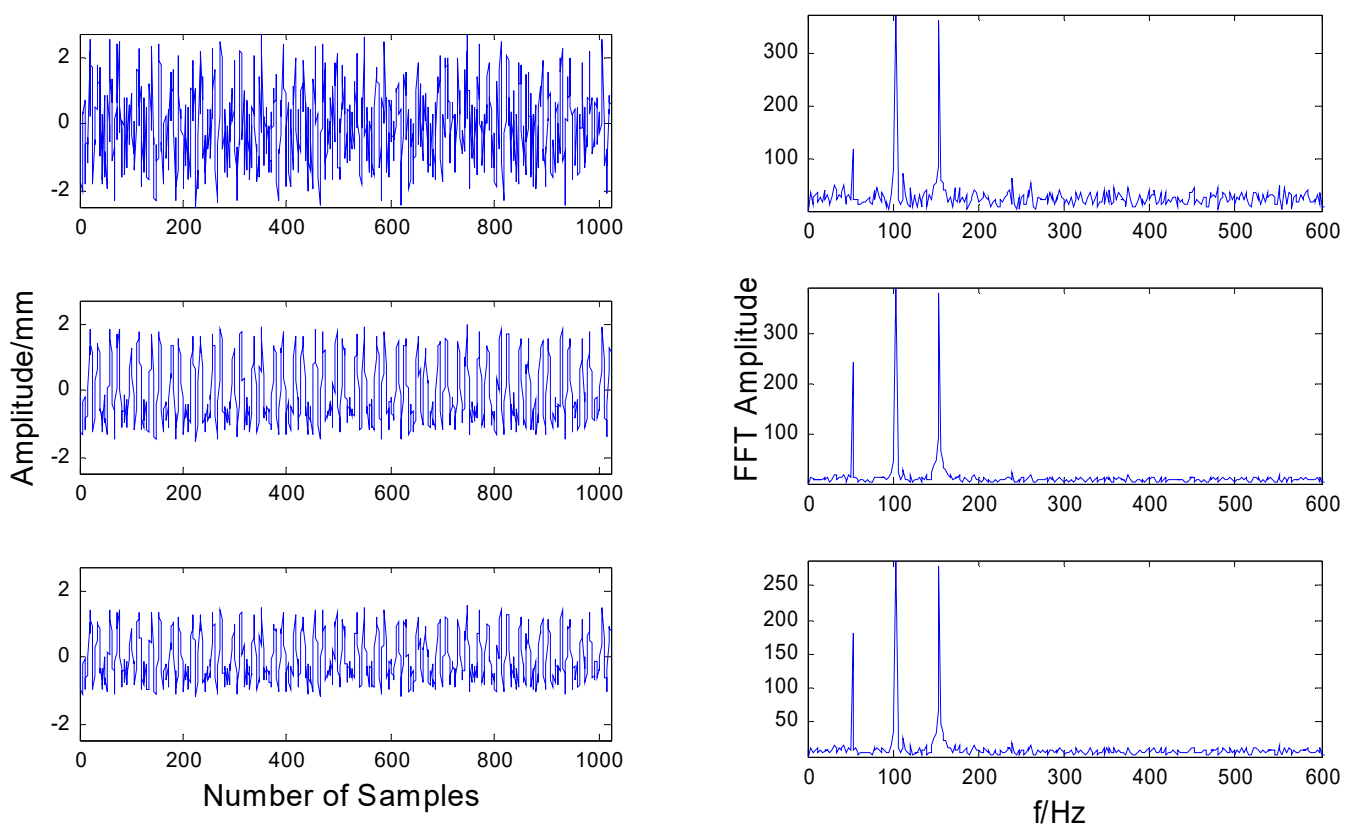

(a) The time domain signals.

(b) The frequency domain signal.

Figure 3. The time-frequency waveform of mixed signal. 
In Figure 3, it can be seen that the characteristics of the source signal have been completely hidden in the mixed signal, and the information on the source signal cannot be read from the time domain waveform.

Figure 4 shows the signal directly separated from the mixed signal by the JADE algorithm. It can be seen, from Figure 4, that the noise signal has not been successfully separated, and the other signals separated contain many harmonics, so the signal characteristics cannot be accurately identified, which indicates that the method is invalid under strong noise.
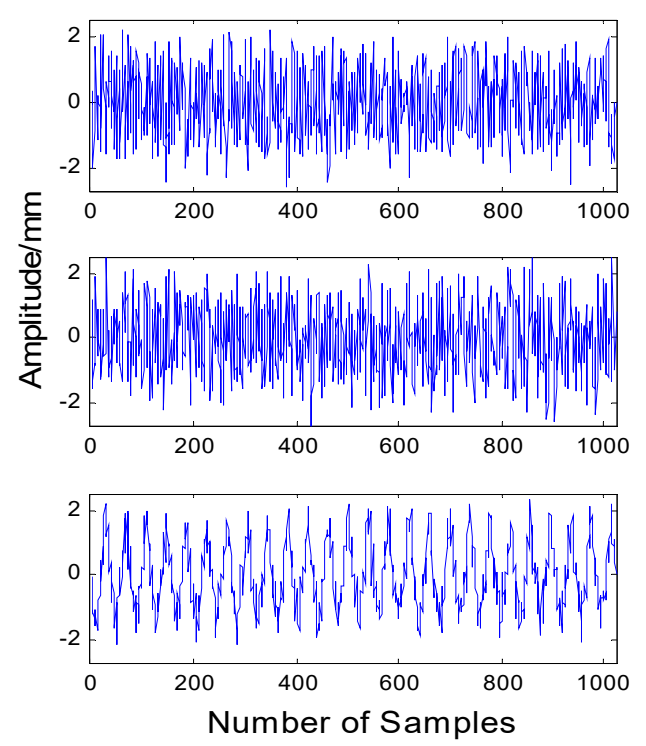

(a) The separated signals with noise by Joint Approximation Diagonalization of Eigenmatrices (JADE)
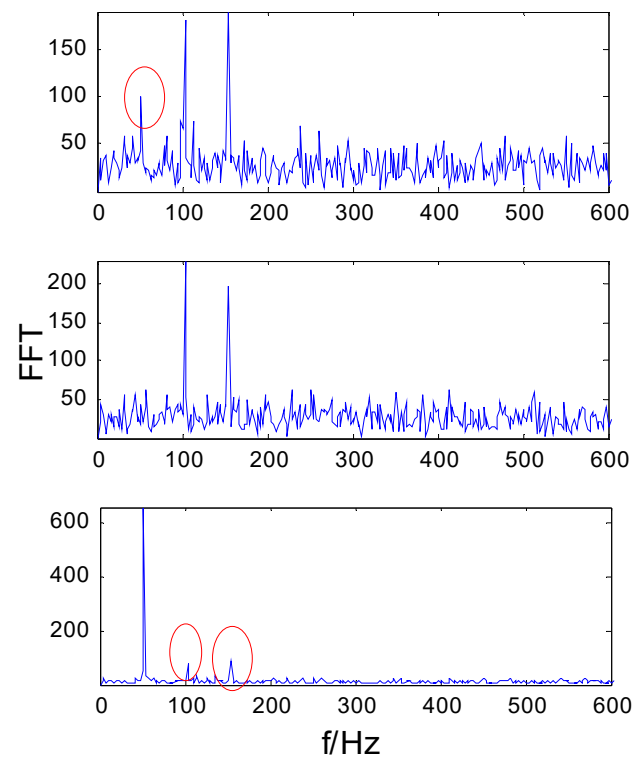

(b) The separated signals with noise by

JADE

Figure 4. The frequency domain signals of the JADE algorithm.

Figure 5 is the signal separated by the classical SOBI algorithm. It can be seen from Figure 5 that, although the noise signal has been successfully separated, the other two separated signals also contain many harmonics, so it is impossible to accurately identify the signal characteristics. It means that this method can separate the noise signal, but it cannot effectively separate other source signals.

By comparing Figures $2 b$ and $4 b$, Figure $5 b$, Figure $6 b$, it can be seen that the separation methods based on the classical JADE and SOBI algorithm have more frequency components marked with red circles. It indicates that the separation result is not accurate. From the evaluation indexes in Table 1 , we can also see that the improved algorithm MF-SOBI is better than SOBI and JADE. 

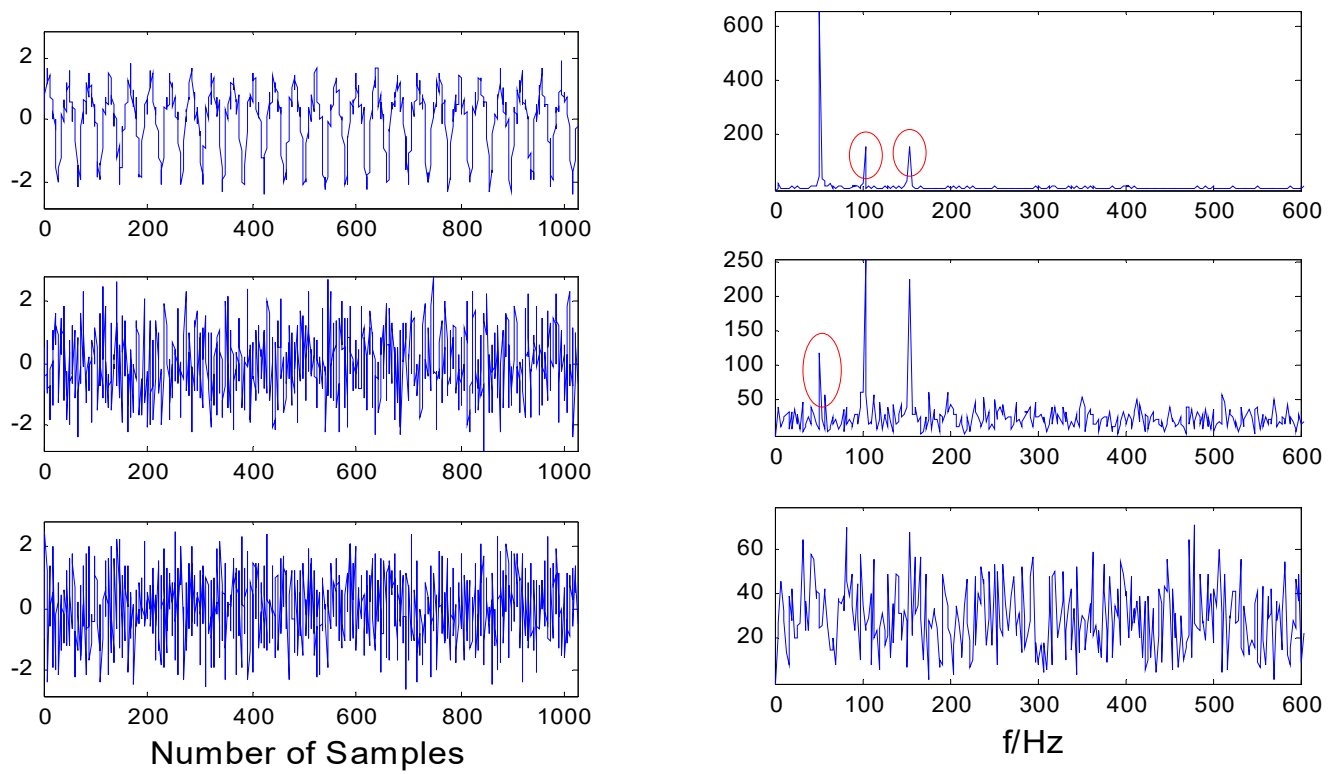

(a) The separated signals with noise by second-order blind identification (SOBI)

(b) The separated signals with noise by SOBI

Figure 5. The frequency domain signals of the SOBI algorithm.
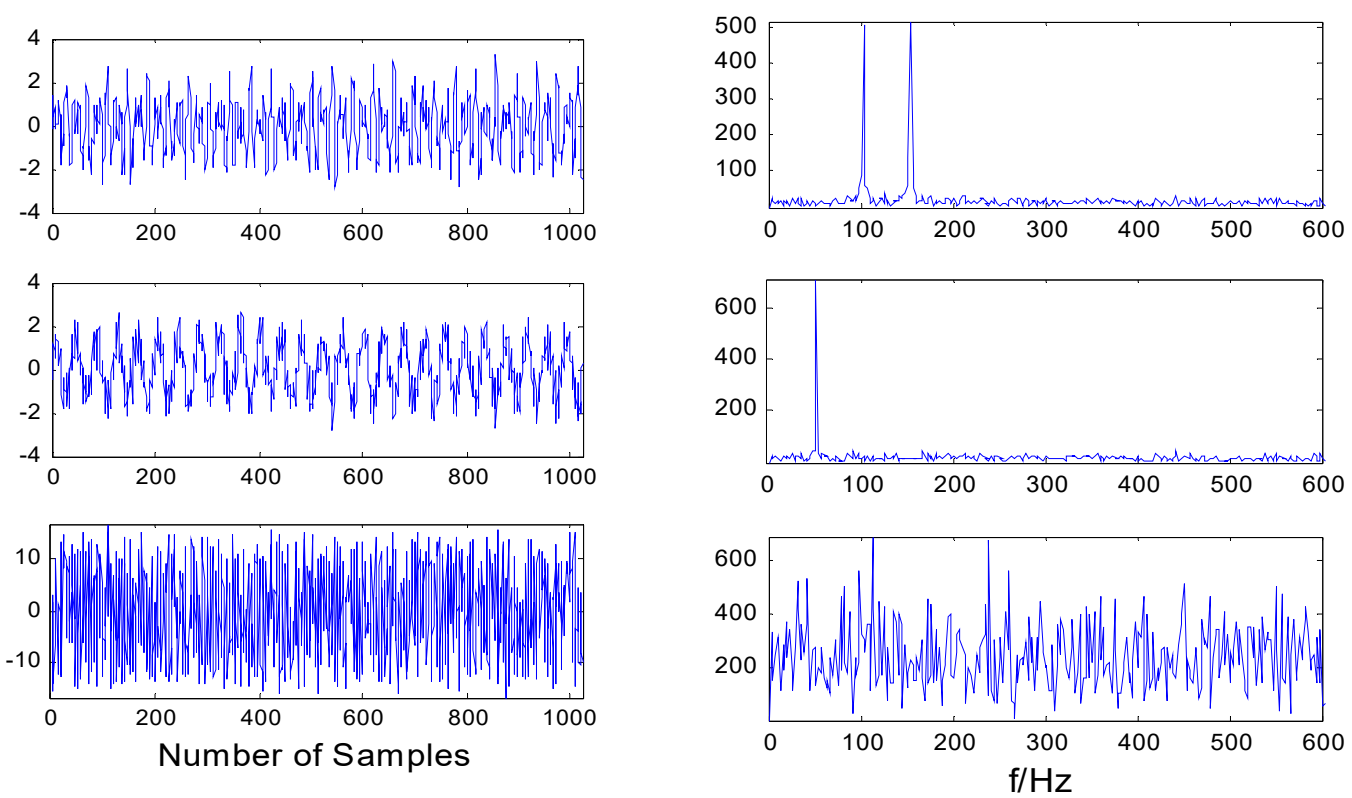

(a) The separated signals with noise by MF-SOBI

(b) The separated signals with noise by MFSOBI

Figure 6. The frequency domain signals of the MF-SOBI algorithm. 
Table 1. Evaluation indexes' comparison of blind source separation.

\begin{tabular}{|c|c|c|c|c|c|}
\hline Algorithm & $s_{i}$ & $\rho_{i}$ & RCTE/dB & PI & $t / s$ \\
\hline \multirow[t]{3}{*}{ JADE } & $s_{1}$ & 0.412 & -2.131 & & \\
\hline & $s_{2}$ & 0.589 & -4.316 & 3.459 & 0.315 \\
\hline & $s_{2}$ & 0.612 & -12.315 & & \\
\hline \multirow[t]{3}{*}{ SOBI } & $s_{1}$ & 0.713 & -12.142 & & \\
\hline & $s_{2}$ & 0.785 & -11.311 & 1.213 & 0.082 \\
\hline & $s_{2}$ & 0.812 & -17.913 & & \\
\hline \multirow[t]{3}{*}{ MF-SOBI } & $s_{1}$ & 0.991 & -23.613 & & \\
\hline & $s_{2}$ & 0.992 & -21.221 & 0.101 & 0.010 \\
\hline & $s_{2}$ & 0.983 & -23.421 & & \\
\hline
\end{tabular}

By comparing Figures 2-6, it can be seen that the uncertainty of the separated signal compared with the source signal mainly lies in the uncertainty of amplitude and signal sequence brought about by the blind source separation technology itself. In addition to this uncertainty, other characteristics of the signal have been better recovered. Furthermore, the performance index and similarity coefficient of the algorithm are calculated and compared with the results of the SOBI and JADE algorithms. The performance index shows the overall separation ability of the algorithm; the smaller the value is, the better. The similarity coefficient shows the reduction of a single source signal; the closer the absolute value is to 1, the better the separation result. The comparison results are shown in Table 1.

From the data in Table 1, it can be seen that, under the interference of strong impulse noise, the improved algorithm in this paper effectively separates the source signals, and has a higher separation performance than the classical algorithm.

\section{Applications}

The double-span rotor experimental platform is shown in Figure 7a. The rotor test bed is $134 \mathrm{~mm}$ long, $51 \mathrm{~mm}$ wide, and $120 \mathrm{~mm}$ high, with a total mass of about $50 \mathrm{~kg}$. The test rotor has a shaft size of $\phi 10 \mathrm{~mm} \times 810 \mathrm{~mm}$, on which three lumped mass disks are installed. The rotor is supported by four cylindrical bearings and separated into a double-span structure. For the convenience of description, the rotor span close to the motor is named the forward span and the rotor span far away from the motor is named the backward span. The rotor system is driven by a direct current (DC) motor whose power is about 1.1 KW. A flexible connection mode of nylon rope is adopted between the motor output shaft and the front span rotor, and between the front span rotor and the rear span rotor. The DC motor is controlled by a pulse width modulator. The experiments show that the first critical speed of the rotor system is about $2000 \mathrm{r} / \mathrm{min}$, the unstable speed is close to $5000 \mathrm{r} / \mathrm{min}$, and $3000 \mathrm{r} / \mathrm{min}$ is the best stable operating point in the flexible working area of the rotor system. The vibration displacement signal acquisition program is implemented on the LabVIEW platform. In order to effectively simulate the rotor imbalance, rotor misalignment, rotor dynamic, and static rub impact fault experiment, the rotor experimental platform is equipped with a counterweight screw, rub impact screw, gasket, and other experimental auxiliary devices. 


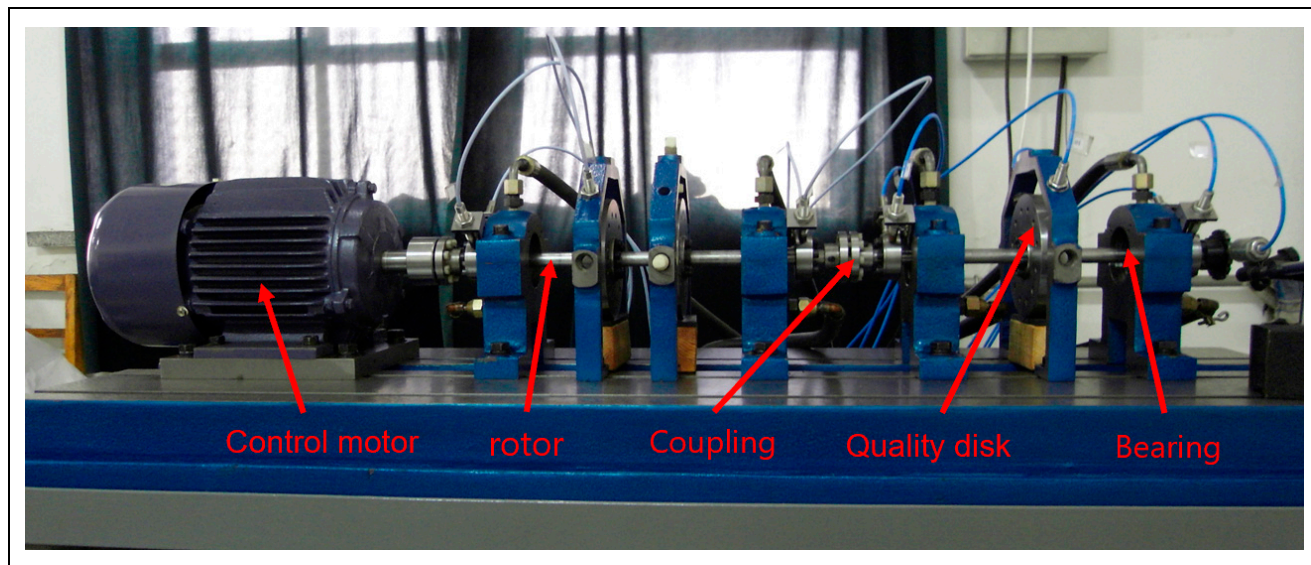

(a) The rotor test-bed

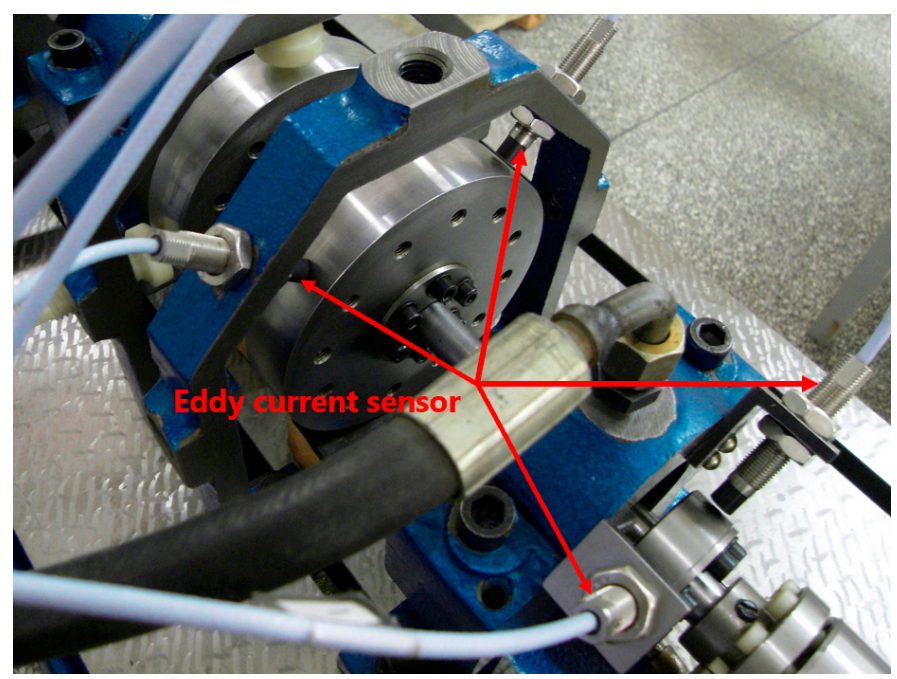

(b) Installation position of sensors

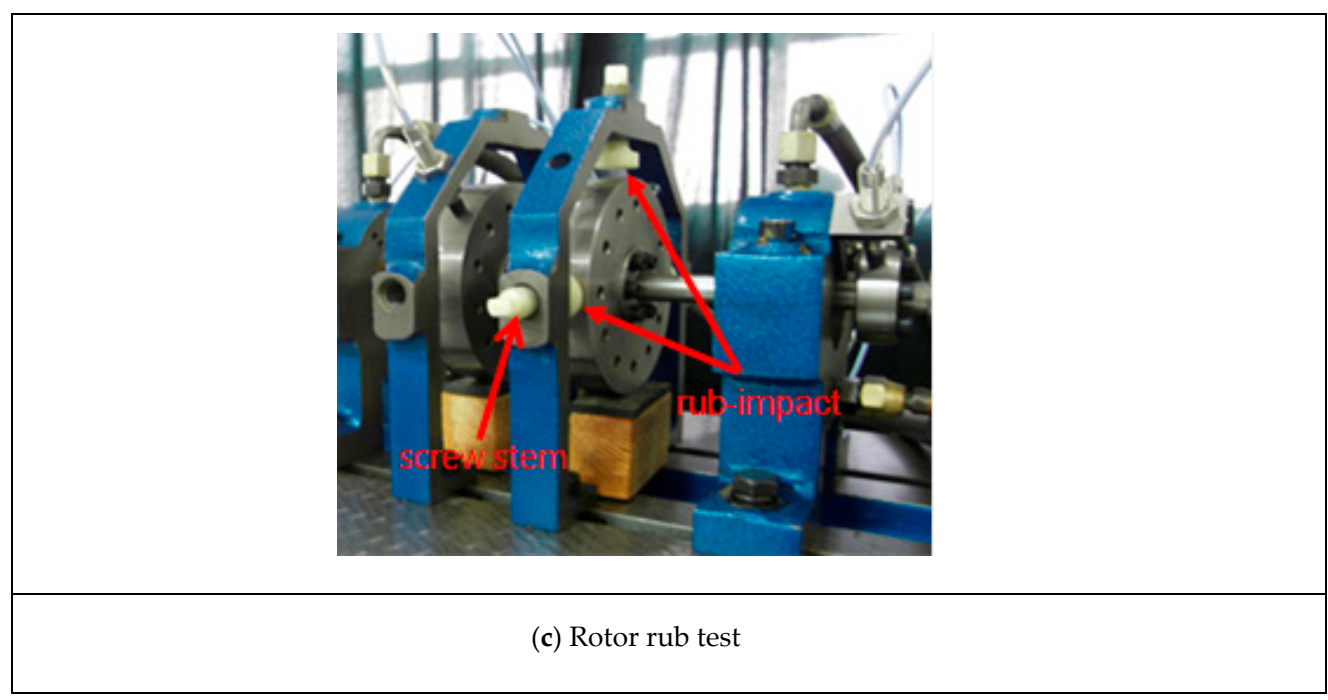

Figure 7. The two-span rotor-bearing rig. 
In order to simulate the rotor dynamic and static rub impact fault on the test bench, a threaded hole is reserved above the test bench bracket, as shown in Figure 7c, and a long white plastic screw is reserved. The long screw is screwed into the threaded hole to make contact with the mass disk, but it cannot make full contact, mainly because, due to the speed increase, the amplitude of the rotor is increasing under the action of unbalanced force. When the amplitude reaches the critical distance, there will be friction between the mass disk and the plastic screw. At the same time, the rub impact fault under different working conditions can be simulated by changing the distance and speed. In this experiment, the dynamic and static rub impact experiments were completed at the speed of $2800 \mathrm{r} / \mathrm{min}$. The sampling frequency was $5000 \mathrm{~Hz}$ and the number of sampling points was 1024 .

The eddy current sensor probe is composed of two vertical probes. The probes are installed near the journal and around the disk. The vibration is obvious and the signal is easy to obtain. The sensor's installation position is shown in Figure $7 \mathrm{~b}$. A single sensor at the end of the rotor is used to measure the real-time speed of the rotor. In order to ensure that the number of sensors in blind source separation is greater than or equal to the number of source signals, four sensors are used in the experiment.

The four measured sensor signals are shown in Figure 8. The signals separated by the SOBI algorithm are shown in Figure 9. The signals separated by the MF-SOBI algorithm are shown in Figure 10.

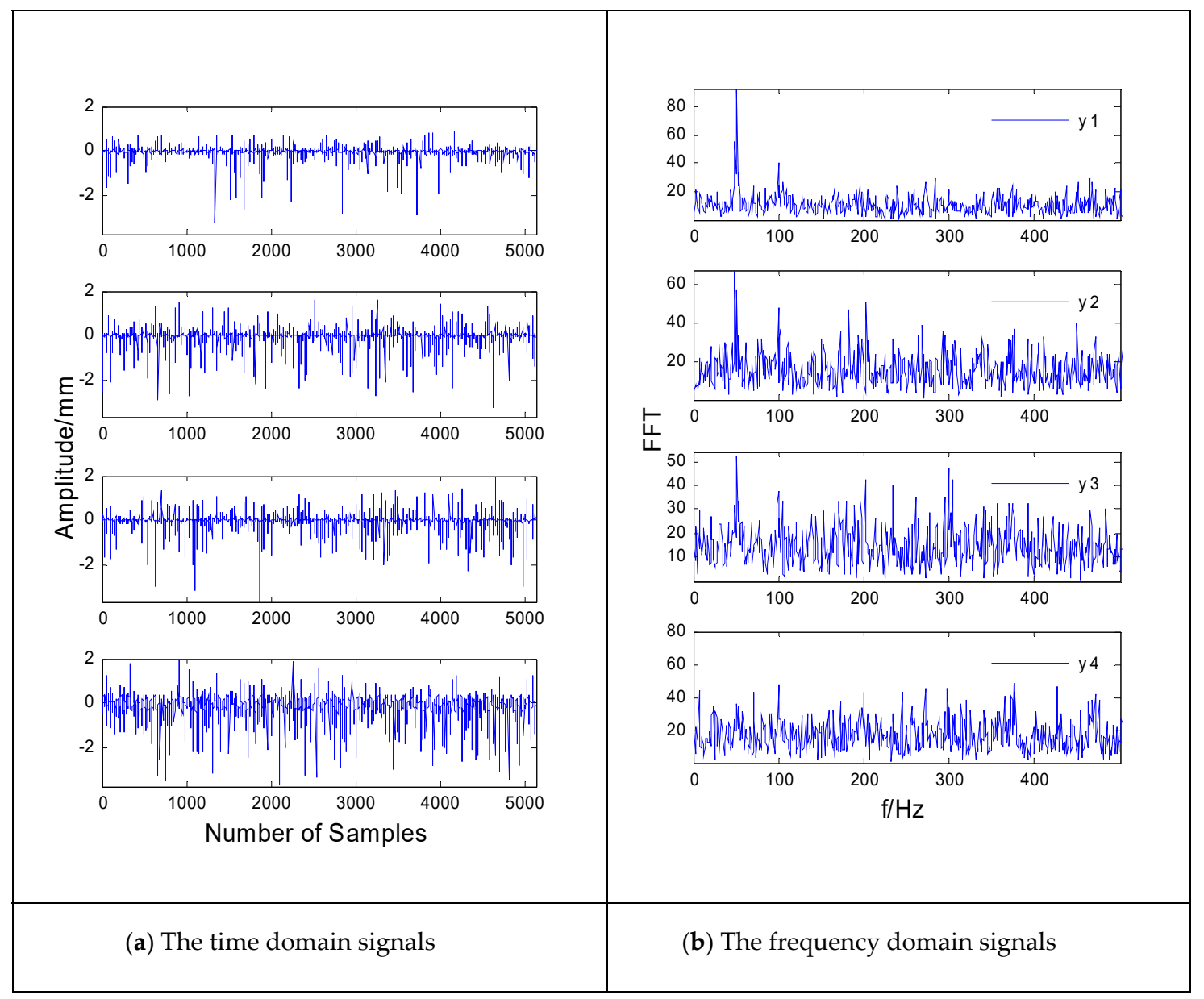

Figure 8. Time-frequency waveforms of the rotor's vibration signals. 


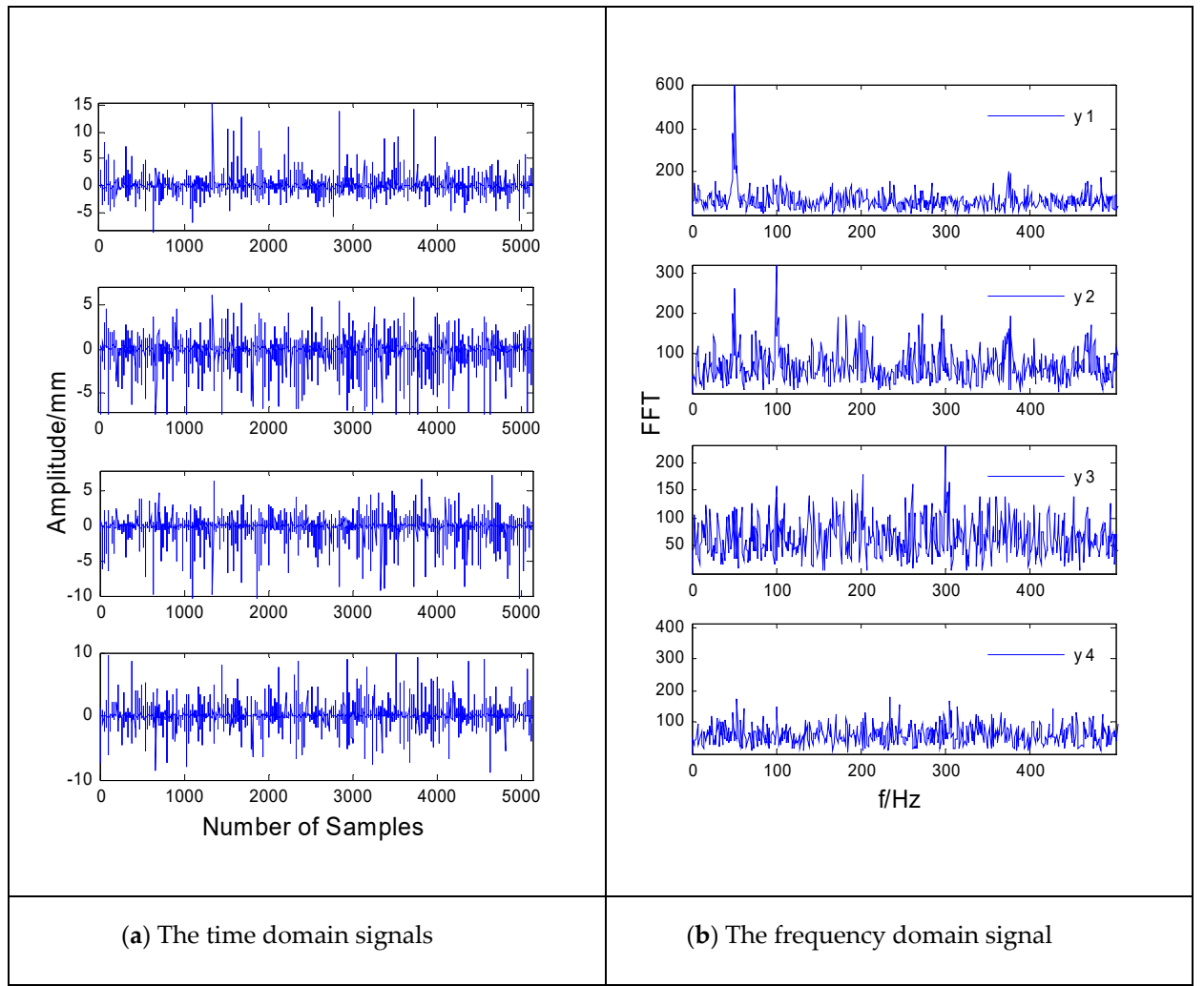

Figure 9. The signals separated by the SOBI algorithm.

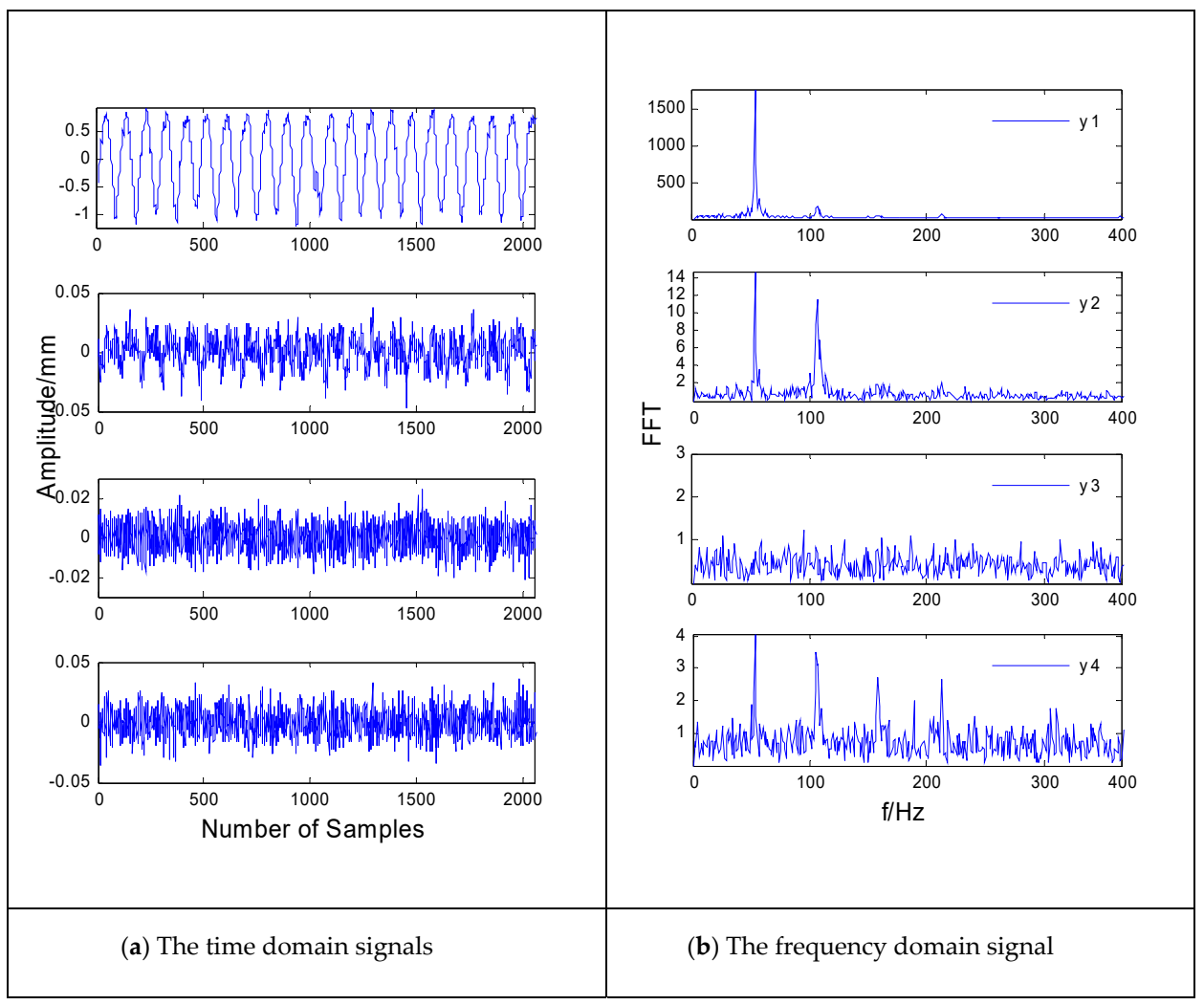

Figure 10. The signals separated by the MF-SOBI algorithm. 
Due to the interference of strong impulse noise, the fault characteristics of the rotor system cannot be identified from the time domain waveform of the source signal in Figure 8a. However, in the frequency domain waveform y1 of Figure $8 b$, the $50 \mathrm{~Hz}$ power frequency signal of the rotor can be clearly identified.

In the case of strong impulse noise, the classical SOBI algorithm is directly used for separation without noise reduction. The separated signal is shown in Figure 9. According to the time-frequency waveform in Figure 9, only a $50 \mathrm{~Hz}$ rotor signal can be clearly identified. This shows that the classical SOBI algorithm has a poor separation effect under strong pulse interference, or is wrong.

As can be seen from Figure 10b (the frequency domain waveform of the separated signal), the impulse noise is well filtered and the rotor vibration source signal is well separated. From y1 of Figure 10, it can be seen that the $50 \mathrm{~Hz}$ signal is obvious, and there is low-amplitude double frequency at the same time. According to the frequency characteristics, it can be determined that the y1 signal clearly reflects the slight imbalance caused by the rub impact fault. From y2 and y4, we can clearly see $50 \mathrm{~Hz}$ power frequency and $100 \mathrm{~Hz}$ double frequency, and a small number of high-frequency components. According to its frequency characteristics, it can be judged as rub impact fault characteristics. Because y3 is random in both the time domain and the frequency domain, it can be judged as random noise.

Through the above analysis, it can be seen that under strong impulse noise interference, the improved algorithm proposed in this paper can effectively separate the rub impact fault of rotor system and the slight imbalance fault caused by the rub impact fault and random noise, which verifies the effectiveness of this method.

\section{Conclusions}

In order to solve the problem of fast fault feature extraction of rotating machinery, an improved blind source separation algorithm based on MF-SOBI is proposed. Our conclusions are as follows:

1. If the observation signal under the interference of strong impulse noise is separated directly, the error of separation result is large, and the wrong result may even be obtained.

2. The improved adaptive median filtering method can effectively remove the interference of strong impulse noise.

3. The improved algorithm in this paper can effectively separate the simulated rotor vibration signals, and its performance is greatly improved compared with the traditional method.

4. The improved algorithm can effectively separate the real rotor vibration signals. By analyzing the time domain and frequency domain of the separated signal, the rotor fault can be inferred, which is consistent with the experimental set-up fault.

Author Contributions: F.M. conceived and designed the experiments; R.Z. provided technical guidance; X.W. and L.J. performed the experiments and analyzed the data; F.M. wrote the paper. All authors have read and agreed to the published version of the manuscript.

Funding: This work was supported in part by the National Natural Science Foundation of China under Grant 51675253, in part by key scientific and technological project of Henan Province under Grant 172102210097, in part by the Foundation of Henan Educational Committee under Grant 16A470021, and in part by Applied science and technology research fund of Luoyang Normal University under Grant 2017-YYJJ-003.

Acknowledgments: I am thankful for the contributions of reviewers and the excellent assistant of the editorial team of Applied Sciences.

Conflicts of Interest: The author declares that there are no conflicts of interest.

\section{References}

1. Meng, Z.; Shi, G.; Wang, F. Vibration response and fault characteristics analysis of gear based on time-varying mesh stiffness. Mech. Mach. Theory 2020, 148, 103786. [CrossRef] 
2. Jiang, X.; Wang, J.; Shi, J.; Shen, C.; Huang, W.; Zhu, Z. A coarse-to-fine decomposing strategy of VMD for extraction of weak repetitive transients in fault diagnosis of rotating machines. Mech. Syst. Signal Process. 2019, 116, 668-692. [CrossRef]

3. Zan, P.; Liu, Y.; Chang, M. Research of rectal dynamic function diagnosis based on FastICA-STFT. IET Sci. Meas. Technol. 2018, 12, 965-969. [CrossRef]

4. Huang, N.E.; Wu, M.-L.; Qu, W.; Long, S.R.; Shen, S.S.P. Applications of Hilbert-Huang transform to non-stationary financial time series analysis. Appl. Stoch. Model. Bus. Ind. 2003, 19, 245-268. [CrossRef]

5. Pan, Z.; Meng, Z.; Chen, Z.; Gao, W.; Shi, Y. A two-stage method based on extreme learning machine for predicting the remaining useful life of rolling-element bearings. Mech. Syst. Signal Process. 2020, 144, 106899. [CrossRef]

6. Abdelkader, R.; Kaddour, A.; Bendiabdellah, A.; Derouiche, Z. Rolling Bearing Fault Diagnosis Based on an Improved Denoising Method Using the Complete Ensemble Empirical Mode Decomposition and the Optimized Thresholding Operation. IEEE Sens. J. 2018, 18, 7166-7172. [CrossRef]

7. Liu, H.; Qin, C.; Liu, M. A Rail Fault Diagnosis Method Based on Quartic C2 Hermite Improved Empirical Mode Decomposition Algorithm. Sensors (Switzerland) 2019, 19, 3300. [CrossRef]

8. McDonald, G.L.; Zhao, Q. Multipoint Optimal Minimum Entropy Deconvolution and Convolution Fix: Application to vibration fault detection. Mech. Syst. Signal Process. 2017, 82, 461-477. [CrossRef]

9. Ma, H.; Feng, Z. Planet bearing fault diagnosis using multipoint Optimal Minimum Entropy Deconvolution Adjusted. J. Sound Vib. 2019, 449, 235-273. [CrossRef]

10. Raja, M.A.Z.; Chaudhary, N.I.; Ahmed, Z.; Rehman, A.U.; Aslam, M.S. A novel application of kernel adaptive filtering algorithms for attenuation of noise interferences. Neural Comput. Appl. 2019, 31, 9221-9240. [CrossRef]

11. Liu, C.; Zhang, Z.; Tang, X. Sign normalised spline adaptive filtering algorithms against impulsive noise. Signal Process. 2018, 148, 234-240. [CrossRef]

12. Li, J.; Li, M.; Zhang, J. Rolling bearing fault diagnosis based on time-delayed feedback monostable stochastic resonance and adaptive minimum entropy deconvolution. J. Sound Vib. 2017, 401, 139-151. [CrossRef]

13. Wang, H.; Sun, D. The application of matching pursuit based on multi feature pattern set in the signal processing of rotating machinery. JVC/J. Vib. Control. 2019, 25, 1974-1987. [CrossRef]

14. Gong, T.; Yuan, X.; Lei, X.; Yuan, Y.; Zhang, B. Fault detection for rolling element bearing based on repeated single-scale morphology and simplified sensitive factor algorithm. Meas. J. Int. Meas. Confed. 2018, 127, 348-355. [CrossRef]

15. De Morais, A.P.; Bretas, A.S.; Brahma, S.; Cardoso, G. High-sensitivity stator fault protection for synchronous generators: A time-domain approach based on mathematical morphology. Int. J. Electr. Power Energy Syst. 2018, 99, 419-425. [CrossRef]

16. Ge, Y. Mathematical Morphology and Deep Learning-based Approach for Bearing Fault Recognition. Int. J. Perform. Eng. 2018, 14, 995. [CrossRef]

17. Sun, R.-B.; Yang, Z.-B.; Gryllias, K.; Chen, X.-F. Cyclostationary modeling for local fault diagnosis of planetary gear vibration signals. J. Sound Vib. 2020, 471, 115175. [CrossRef]

18. Mauricio, A.; Qi, J.; Gryllias, K. Vibration-Based Condition Monitoring of Wind Turbine Gearboxes Based on Cyclostationary Analysis. J. Eng. Gas Turbines Power 2018, 141, 031026. [CrossRef]

19. Yu, L.; Antoni, J.; Wu, H.; Jiang, W. Reconstruction of cyclostationary sound source based on a back-propagating cyclic wiener filter. J. Sound Vib. 2019, 442, 787-799. [CrossRef]

20. Cheng, C.; Wang, W.; Luo, H.; Zhang, B.; Cheng, G.; Teng, W. State-Degradation-Oriented Fault Diagnosis for High-Speed Train Running Gears System. Sensors (Switzerland) 2020, 20, 1017. [CrossRef]

21. Bayrak, G. Wavelet transform-based fault detection method for hydrogen energy-based distributed generators. Int. J. Hydrogen Energy 2018, 43, 20293-20308. [CrossRef]

22. Özmen, G.; Özşen, S. A new denoising method for fMRI based on weighted three-dimensional wavelet transform. Neural Comput. Appl. 2017, 29, 263-276. [CrossRef]

23. Xin, Y.; Li, S.; Wang, J.; Yi, P.; Liu, J. Gear fault diagnosis method based on iterative empirical wavelet transform. Yi Qi Yi Biao Xue Bao/Chin. J. Sci. Instrum. 2018, 39, 79-86.

24. Kang, Y.; Shi, Z.; Zhang, H.; Zhen, D.; Gu, F. A Novel Method for the Dynamic Coefficients Identification of Journal Bearings Using Kalman Filter. Sensors (Switzerland) 2020, 20, 565. [CrossRef] 
25. Pan, M.-C.; Chu, W.-C.; Le, D.-D. Adaptive angular-velocity Vold-Kalman filter order tracking-Theoretical basis, numerical implementation and parameter investigation. Mech. Syst. Signal Process. 2016, 81, $148-161$. [CrossRef]

26. Shi, P.; Su, C.; Han, D. Fault Diagnosis of Rotating Machinery Based on Adaptive Stochastic Resonance and AMD-EEMD. Shock. Vib. 2016, 2016, 1-11. [CrossRef]

27. Lu, S.; He, Q.; Dai, D.; Kong, F. Periodic fault signal enhancement in rotating machine vibrations via stochastic resonance. J. Vib. Control. 2016, 22, 4227-4246. [CrossRef]

28. Fu, W.; Tan, J.; Zhang, X.; Chen, T.; Wang, K. Blind Parameter Identification of MAR Model and Mutation Hybrid GWO-SCA Optimized SVM for Fault Diagnosis of Rotating Machinery. Complexity 2019, 2019, 1-17. [CrossRef]

29. Zhang, T.; Zhang, H.; Liu, D.; Li, Q. Frequency Domain Blind Source Separation Permutation Algorithm Based on Regional Growth Correction. Dianzi Yu Xinxi Xuebao/J. Electron. Inf. Technol. 2019, 41, 580-587.

30. Fantinato, D.G.; Duarte, L.T.; Deville, Y.; Attux, R.; Jutten, C.; Neves, A.O. A second-order statistics method for blind source separation in post-nonlinear mixtures. Signal Process. 2019, 155, 63-72. [CrossRef]

31. Lee, J.-H.; Kwon, H.-J.; Jin, Y.-K. Numerically Efficient Implementation of JADE ML Algorithm. J. Electromagn. Waves Appl. 2008, 22, 1693-1704. [CrossRef]

32. Li, Z.; Peng, Z. A new nonlinear blind source separation method with chaos indicators for decoupling diagnosis of hybrid failures: A marine propulsion gearbox case with a large speed variation. Chaos Solitons Fractals 2016, 89, 27-39. [CrossRef]

33. Miao, F.; Zhao, R.; Wang, X. Research on the Fault Feature Extraction Method of Rotor Systems Based on GAW-PSO. Math. Probl. Eng. 2020, 2020, 1-10. [CrossRef]

34. Grotas, S.; Yakoby, Y.; Gera, I.; Routtenberg, T. Power Systems Topology and State Estimation by Graph Blind Source Separation. IEEE Trans. Signal Process. 2019, 67, 2036-2051. [CrossRef]

35. Huang, J.; Sun, J. Sampling Adaptive Learning Algorithm for Mobile Blind Source Separation. Wirel. Commun. Mob. Comput. 2018, 2018, 1-7. [CrossRef]

36. Wang, C.-C.; Zeng, Y.-H. Research Status and Prospects of Underdetermined Blind Source Separation Algorithms. Beijing Youdian Daxue Xuebao/J. Beijing Univ. Posts Telecommun. 2018, 41, 103-109.

37. Miao, F.; Zhao, R.; Wang, X.; Jia, L. A New Fault Feature Extraction Method for Rotating Machinery Based on Multiple Sensors. Sensors 2020, 20, 1713. [CrossRef]

38. Belaid, S.; Hattay, J.; Naanaa, W.; Aguili, T. A new multi-scale framework for convolutive blind source separation. Signal Image Video Process. 2016, 10, 1203-1210. [CrossRef]

39. He, C.; Li, H.; Zhao, X. Weak characteristic determination for blade crack of centrifugal compressors based on underdetermined blind source separation. Meas. J. Int. Meas. Confed. 2018, 128, 545-557. [CrossRef]

40. Miao, F.; Zhao, R. A New Fault Diagnosis Method for Rotating Machinery Based on SCA-FastICA. Math. Probl. Eng. 2020, 2020, 1-12.

41. Li, L.; Qu, L. Second-order cyclic statistics for mechanical fault diagnosis. Hsi-An Chiao Tung Ta Hsueh/J. Xi'an Jiaotong Univ. 2002, 36, 943-946.

42. Ming, Y.; Chen, J.; Dong, G. Application of convolved blind separation based on second-order cyclic statistics in rolling element bearing feature extraction. J. Vib. Control. 2012, 20, 617-633. [CrossRef]

43. Borghesani, P.; Pennacchi, P.; Ricci, R.; Chatterton, S. Testing second order cyclostationarity in the squared envelope spectrum of non-white vibration signals. Mech. Syst. Signal Process. 2013, 40, 38-55. [CrossRef]

44. McCormick, A.; Nandi, A. Cyclostationarity in rotating machine vibrations. Mech. Syst. Signal Process. 1998, 12, 225-242. [CrossRef]

45. Xin, G.; Hamzaoui, N.; Antoni, J. Extraction of second-order cyclostationary sources by matching instantaneous power spectrum with stochastic model-Application to wind turbine gearbox. Renew. Energy 2020, 147, 1739-1758. [CrossRef]

46. Xi, J.; Cui, J.; Jiang, L. JADE-ICA-based blind source separation of multi-fault signals of rolling bearings. Zhendong yu Chongji/J. Vib. Shock 2017, 36, 231-237.

(C) 2020 by the authors. Licensee MDPI, Basel, Switzerland. This article is an open access article distributed under the terms and conditions of the Creative Commons Attribution (CC BY) license (http://creativecommons.org/licenses/by/4.0/). 